Volume 9, No.4, July - August 2020

International Journal of Advanced Trends in Computer Science and Engineering

Available Online at http://www.warse.org/IJATCSE/static/pdf/file/ijatcse112942020.pdf

https://doi.org/10.30534/ijatese/2020/112942020

\title{
A Plant Disease Detection and Classification using Image Segmentation Technique
}

\author{
D.Angayarkanni ${ }^{* 1}$, Dr.L.Jayasimman ${ }^{2}$ \\ ${ }^{1}$ Research Scholar, Department of Computer Science, Bishop Heber College (Autonomous), \\ Affiliated to Bharathidasan University, Trichy, Tamilnadu, India. \\ ${ }^{2}$ Assistant Professor, Department of Computer Science, Bishop Heber College(Autonomous), \\ Affiliated to Bharathidasan University, Trichy, Tamilnadu, India
}

\begin{abstract}
Our economy highly depends on Agricultural production. Leaf disease identification in plants plays an significant role in the agricultural sector. In the age of technology, automation is the best solution to any worry. The performance of the leaf disease detection largely depends on the segmentation result of diseased part of the image. So, Image segmentation plays an important role in identification of diseased parts and for further analysis such as feature extraction and classification. This work proposes an automatic detection of plant leaf disease using image segmentation based on color transformation and thresholding. Color, Gray Level Co-occurrence Matrix(GLCM), Local Binary Pattern(LBP) characteristics are taken from the segmented image and Support Vector Machine(SVM), KNN classification technique used to classify the diseased part. The segmentation part with the combination of color, GLCM, LBP features gives better accuracy with SVM technique.
\end{abstract}

Key words:-Image segmentation, Feature Extraction, GLCM, LBP, SVM

\section{INTRODUCTION}

The maximum food crops are affected by lot of diseases through environmental conditions and are malnourished. This may leads to huge loss of the farmers which may reduce to take proper care with expert's advice. The problem can be solved with the help of technological advancement. "IMAGE PROCESSING" is such a solution for detection and resolution of this problem. This study focuses on the development of a leaf disease automatic detection and classification algorithm for plant diseases. This works consists of four phases such as pre-processing, Segmentation, Feature Extraction and Classification. The identification and classification of disease in leaves of plants depends on Image segmentation and feature extraction techniques. Image segmentation divides a picture into many segments or sections .It is also used to locate objects and boundaries
[1].Generally, Image segmentation techniques is broadly classified into four types such as Thresholding, clustering Based, Region Based and Edge Based methods. Thresholding based Method uses histogram of an image to find single or multiple threshold. In Region Based segmentation, pixels are segmented on their similarity value. In clustering based method, Pixels are grouped according to their features like color, texture and shape. In edge based method, pixels are grouped according to the discontinuity value of pixels. Features are extracted from the image to classify plant disease. They are given as an input to the disease identification classification. The color, histogram, texture and shape are characteristics of an image. The GLCM, LBP, etc, are different texture characteristics derived from images. Such characteristics form a vector and are provided as an input to the classification purpose. The algorithms of classification are classified according to regulated and unattended techniques. The SVM (Support Vector Machine), K- the next closest neighbor classifiers, Artificial Neural Networks, and Fuzzy Logy Rabia Masood S.A et al.[2] are used to identify the classifiers. In this research supervised technique is used for classification. For experimental purpose fungal disease of Potato leaf images such as early blight and late blight are taken.

\section{RELATED WORK}

Ramesh Babu et al.[3] proposed a K-means clustering for image segmentation and GLCM for feature extraction and KNN for classification of plant leaf diseases. They achieves classification accuracy between 70 to $75 \%$ for different inputs. Samiksha Devi et al. [4] uses GLCM and LBP feature extraction, k-means for image segmentation and SVM classification for Plant disease identification. Ganesan et al.[5] performed plant leaf analysis using Fuzzy based Segmentation on CIELUV color space and analysis the segmented image using various image quality parameters in GLCM. Piyush Chaudhary et al. [6] performed disease Spot Detection on Plant Leaf using various color spaces such as YCBCR, HSI and CIELAB. OTSU Method is used for thresholding for segmentation of the image and comparison is done with all these color spaces. HSI, RGB, YCbCr and CIELAB color models. 
Neelaveni, et al.[7] and Radhika Deshmukh et al.[8] used k-means clustering and neural network classifier for detection and classification of paddy leaf diseases. Joshi et al.[9] performed rice leaf disease classification using YCBCR color image segmentation, color and shape features are given as an input to MDC and k-NN classifier. Pranjali B. Padol et al.[10] proposed SVM based classifier for detecting and identifying grape leaf diseases using k-means clustering for image segmentation and GLCM and LBP based features. Prabira Kumar Sethy et al.[11] proposed two series of SVM classifier for detection and identification of rice plant diseases with PSO.

Hema et al. [12] proposed an interactive development tool that segment the foreground from an image by using the values for H (Hue), S (Saturation) and V (Value). Ganesan et al.[13] proposed a segmentation of ROI from satellite images using HSV color space.

Garcia-Lamont et al.[14] proposed a color image segmentation method by training SOM using chromaticity features of different colors. For chromaticity, HSV color space is used because in HSV, Chromaticity is decoupled from intensity.R. Amirulah et al.[15] proposed a segmentation technique based on YCBCR color space for starfruit from the image. Khenilyn P. Lewis et al.[16] studied and performed the image processing techniques and data mining algorithms to classify the coffee palnts leaves. Vikram R et al.[17] proposed an efficient traffic control system using pattern matching algorithm in image processing technique. A.Joel Dickson et al. [18] studied and analyzed the image segmentaion algorithm to detect the liver disorder using the nature of user interaction.

\section{PROPOSED METHODOLOGY}

\section{Algorithm-1 Improved Leaf Detection Algorithm} (ILDA)

Step 1: Input Image

Step 2: Resizing an Image into [256X256] Image.

Step 3: Perform Median Filtering to remove noise from the image.

Step 4: Perform Contrast Stretching to enhance the Image.

Step 5: Image segmentation using color transformation and FCM clustering techniques.

5.1 Convert RGB into YCBCR color space

5.2 Obtain foreground binary Image using FCM and $\mathrm{CB}, \mathrm{CR}$ color component.

5.3 Convert into to RGB.

5.4 Convert the result image into HSV.

5.5 Create a mask to extract healthy and diseased part.

Step 6: Extract GLCM and LBPH features from the segmented diseased part of the image.

Step 7: Train the features using SVM.

Step 8: Test and Classify the Leaf Disease.

Algorithm-1 explains the process of detection of disease in a plant leaf using an image segmentation technique. The phases of classification used in the proposed algorithm are shown below.

\subsection{Image Pre-processing}

The goal of image pre-processing is to suppress the noise present in the image and to enhance the details of the image. Image preprocessing is performed in 2 stages. In the first stage noise removal is performed and then image is enhanced. In this work, Median filter is used for noise removal It is a nonlinear filter, which is used to eliminate salt which pepper noise in particular. The output images are obtained by sliding over the image through a $3 \times 3$ window. The middle position of the window is replaced by the input window median of the values. Contrast Stretching is a technique for enhancing photos. The picture is enhanced by expanding the range of intensity values it contains to completely exploit potential values.

\subsection{Image Segmentation}

To segment diseased part from the Image, background should be removed from the Image. Foreground separation is performed to transform the RGB image into YCBCR and HSV Color Space. Color Models also called Color Spaces which decribes the ways of showing human color vision system modeled. It is an abstract mathematical model that displays colors are represented as color components and it is a basic form image representation. There are different color space like RGB, YCbCr, HSV, CMY, CMYK and HSI. In RGB,the colors are represented as three color components R,G,B.Each pixel in RGB image contains stack of 3 layers such as R,G,B.HSV is very close to RGB. HSV color space is

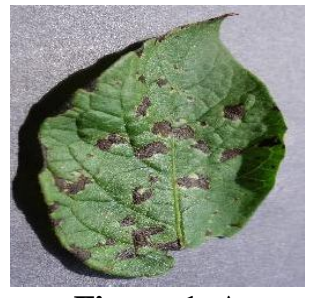

Figure 1. A

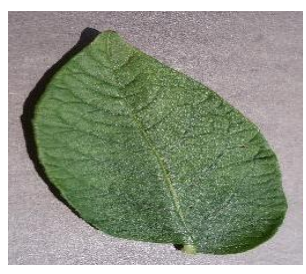

Figure 2.A

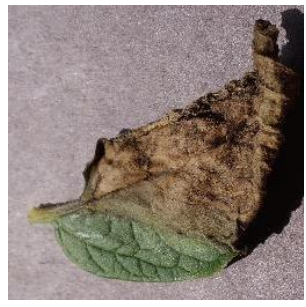

Figure 3.A

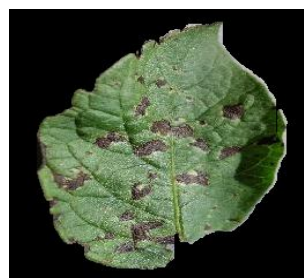

Figure 1.B

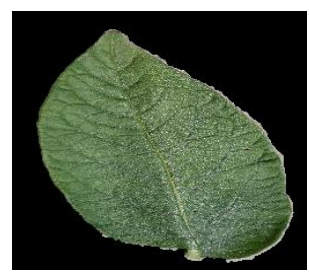

Figure 2.B

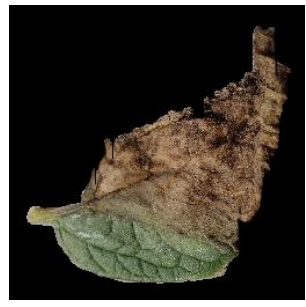

Figure 3.B
Figure 1:A,2.A,3.A - Original Image and Figure 1.B,2.B,3.B - Forground Image 
represented as hexacone in which central axis represents intensity. It consists of 3 color components Hue H,Saturation $\mathrm{S}$ and Value V.Hue represents shades of color. The saturation defines the purity of the color or hue i.e., what amount of white color is mixed with hue. The value reflects the colour's brightness. Saturation (S)[ is the distance from the central Axis of HSV ring. Hue $(\mathrm{H})$ starts at the primary red at $0^{\circ}$ and goes back to primary green 120 o and primary blue $240^{\circ}$ and then back to red at $360^{\circ}$. You can separate $\mathrm{YCbCr}$ from luminance components (Y) and two chrominance components ( $\mathrm{Cd}$ and $\mathrm{Cr}$ ) separately. You can also select $\mathrm{YCbCr}$ as color brightness, and the signals $\mathrm{Cb}$ and two color difference $\mathrm{CB}$ and $\mathrm{B}(\mathrm{B}-\mathrm{Y})$ and the color red minus luma (R-Y).

Diseased part and healthy part is segmented with $h \& S$ component of HSV Color space.

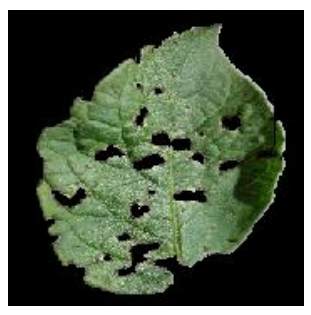

Figure 4.A

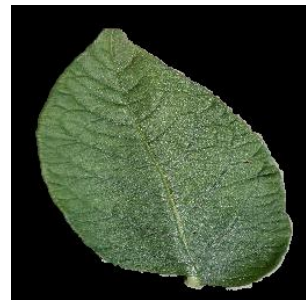

Figure 5.A

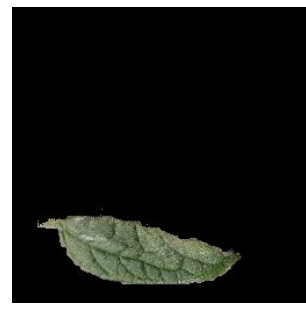

Figure 6.A

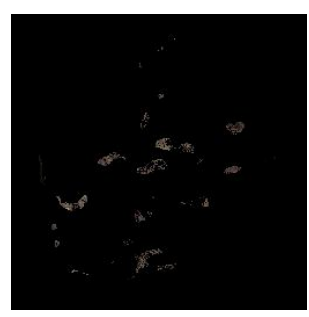

Figure 4.B

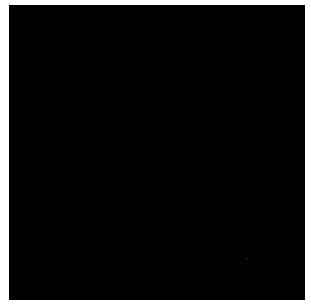

Figure 5.B

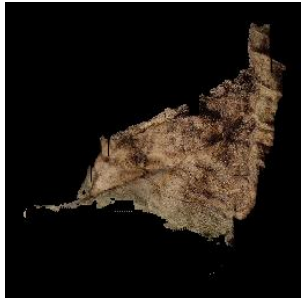

Figure 6.B

\subsection{Fuzzy C means Algorithm}

Theory of Fuzzy and Fuzzy logic. Objects in FCM clustering are part of more than one class. Every entity belongs to every cluster with a certain weight. Assume $n$ objects set $X=\{x 1, x 2, \ldots, x n\}$ where $x i$ is a d-dimensional point. A fuzzy cluster comprises a group of k clusters, C1,C2, $\ldots, \mathrm{Ck}$ and $\mathrm{W}=$ wi, $\mathrm{j}[0,1]$ and $\mathrm{I}=1 \ldots \ldots \mathrm{N} \& \mathrm{j}=1 \ldots \mathrm{k}$, where every wi element, is the weight of the object I in the $\mathrm{Cj}$ cluster. $\underset{i \leq j \leq K}{\forall} C_{j}=\frac{\sum_{i=1}^{n}\left(W_{i j}\right)^{p} x_{i}}{\sum_{i=1}^{n}\left(W_{i j}\right)^{p}}$

The objective function of $\mathrm{fcm}$ is to minimize

$\arg \min \sum_{i=1}^{n} \sum_{j=1}^{k}\left(W_{i j}\right)^{p}\left\|x_{i}-c_{j}\right\|^{2}$

fuzzy membership function

$$
W i j=\frac{1}{\sum_{k=1}^{c}\left(\frac{\left\|x_{i}-c_{j}\right\|}{\left\|x_{i}-c_{k}\right\|}\right)^{\frac{2}{p-1}}}
$$

Figure 4.A,5.A,6.A shows the healthy part of image and Figure 4.B,5.B,6.B displays the diseased part of the image.

\subsection{Feature Extraction}

The feature removal process is useful if the amount of resources available for processing is to be decreased without any substantial or important information being lost . Function extraction increases the precision of learned models by extracting input features. Generally, Color, texture and shape features are extracted from the images. In this work, color features , texture features such as GLCM and LBP are chosen for extracting features from the leaf images.

\subsection{Color and Histogram features}

In RGB image, all the three components are separated and the overall mean values of each component are computed. Next from gray scale histogram, no. of pixels of each intensity is derived and the feature vector is formed.

\subsection{Gray Level Co-occurrence Matrix (GLCM)}

GLCM is a gray level Co-occurrence Matrix that determines the pixel-spatial relationship in the image. Second order statistical characteristics are derived using GLCM. A GLCM is a matrix, in which the number of rows and columns in the image is equal to the number of gray levels. GLCM indicates how often each gray level occurs at a pixel in a fixed geometric location relative to any other pixel. It consists of two steps for extraction of the function. In the first step, the GLCM is computed while the GLCM-based texture features are determined in the second. The GLCM features are: Contrast, Correlation, Energy, Homogeneity, Mean, Standard Deviation, Entropy, RMS, Variance, Smoothness, Kurtosis, Skewness and Inverse Difference Moment.

\subsection{Local Binary Pattern (LBP)}

The Local binary pattern operator is an image operator that converts an image into an integer label array or expression that defines image textures. LBP label statics are used for extraction of functionality. The Histogram (LBPH) functions are derived from the LBP Mark. For the calculation of the LBP code, the 8 central pixel neighbors (xi, $\mathrm{i}=1: 8$ ) are compared to the central pixel(p) for each pixel $\mathrm{p}$ and the neighbors are assigned a value 1 if $\mathrm{xi} p$ is otherwise zero. This phase is measured over the entire picture. From this label, Histogram is formed. This feature is used for classification purpose.

All the above features are computed and they are combined to form a new feature vector for classification of disease. 


\subsection{Classification}

For classifying pixels in the image, supervised machine Learning Method SVM is used. SVM is a linear model used for both classification and regression problem. The main goal of SVM is to separate the datasets into classes for finding a maximum marginal hyperplane. This classification works by finding the optimum hyper-plane that differentiates the two classes very well.

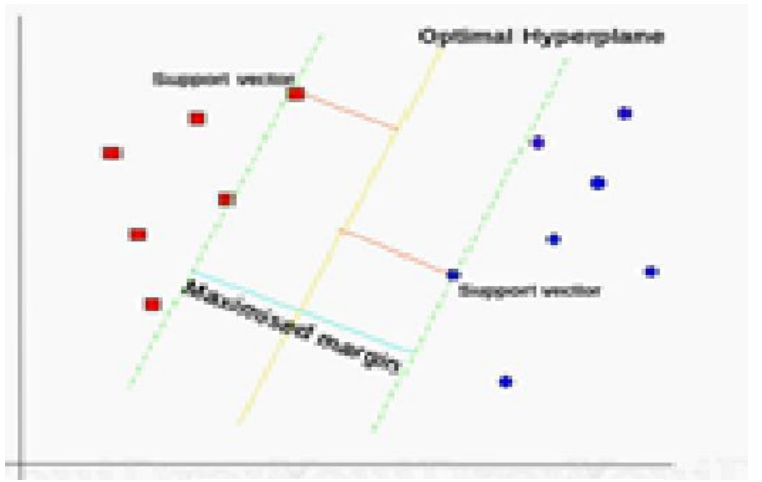

Figure 7: Hyperplane with SVM

In multiclass problem, several binary classifiers are combined to solve the problem. It uses ONE vs -ALL approach constructs $\mathrm{k}$ SVM models where $\mathrm{k}$ is the number of classes in which $\mathrm{m}^{\text {th }} \mathrm{SVM}$ is trained with all the examples with positive labels in the $\mathrm{m}^{\text {th }}$ class, and all other examples with negative labels. One vs-one method divides the dataset into one class and another class in one dataset..

\section{Result and Discussions}

The samples of plant diseased leaves are collected from Plant Village Dataset. This dataset consists of leaf disease for various plants. For experimental purpose potato leaves were taken. It consists of 3 types of images with 2 diseases such as Early Blight, Late Blight and healthy leaf Images. Implementation is done with MATLAB R2016a. 259 features of color, 13 features of GLCM and 256 features of the LBP histogram are taken. They are given as input to SVM and KNN technique. Accuracy of KNN and ILDA with SVM classifier is computed with various feature extraction techniques.

Table 1: Classifier Accuracy

\begin{tabular}{|c|c|c|c|}
\hline S.no & $\begin{array}{c}\text { Extraction } \\
\text { Techniques }\end{array}$ & KNN & $\begin{array}{c}\text { ILDA(Propo } \\
\text { sed) }\end{array}$ \\
\hline 1 & GLCM & $72.0 \%$ & $77.8 \%$ \\
\hline 2 & LBPH & $69.7 \%$ & $82.0 \%$ \\
\hline 3 & COLOR & $78.2 \%$ & $83.0 \%$ \\
\hline 4 & GLCM+LBPH & $87.3 \%$ & $89.0 \%$ \\
\hline 5 & $\begin{array}{c}\text { GLCM+LBPH+CO } \\
\text { LOR }\end{array}$ & $88.8 \%$ & $89.7 \%$ \\
\hline
\end{tabular}

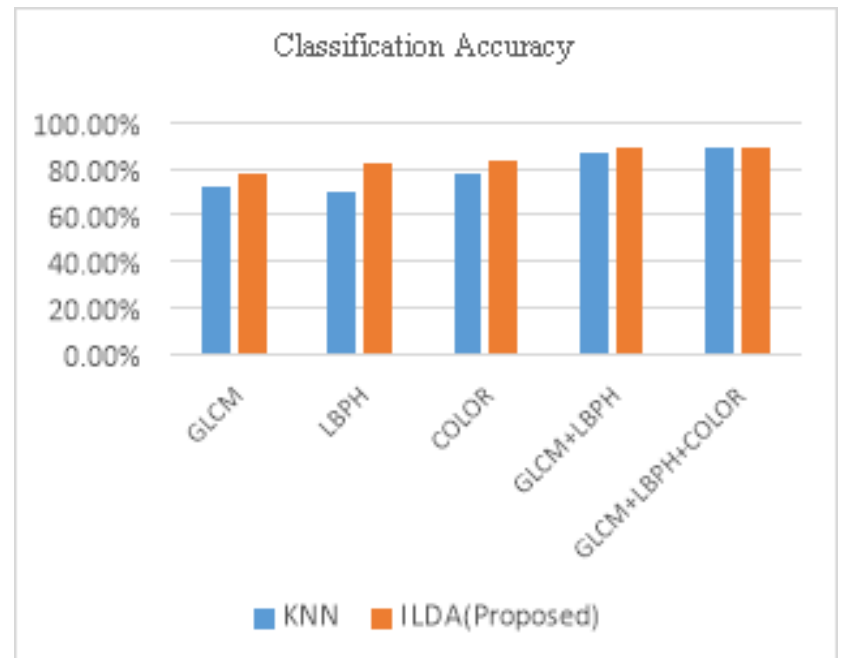

Figure 8:- Classification Accuracy

\section{CONCLUSION}

Leaf Disease Detection and classification highly depends on segmentation and features used for classification. In this work, YCBCR and HSV color space transformation is used for segmentation. Various features such as color ,texture are extracted from the segmented portion and it is given as input to KNN and SVM classifier. Combination of color and texture features LBPH and GLCM give higher accuracy than others. In future, various leaves will be taken for experiment and different segmentation and feature extraction method will be used for higher classification accuracy.

\section{REFERENCES}

[1].What is image segmentation Available at: https://en.wikipedia.org/wiki/Image_segmentaion.

[2]. Rabia Masood, S.A. Khan, M.N.A. Khan, Plants Disease Segmentation using Image Processing, International Journal of Modern Education and Computer Science, volume 8, Issue 1, 2016, pp. 24-32.

https://doi.org/10.5815/ijmecs.2016.01.04

.[3] [3]. Ch Ramesh Babu, Dammavalam Srinivasa Rao, V. Sravan Kiran, N. Rajasekhar, Assessment of Plant Disease Identification using GLCM and KNN Algorithms, International Journal of Recent Technology and Engineering (IJRTE), Volume-8 Issue-5, January 2020..

[4] Samiksha Devi, Ritika, Bhanu Gupta, GLCM-LBP Plant Leaf Disease Detection, International Journal of Scientific Research and Engineering Development_Volume 2 Issue 3, June 2019 pp.136-140.

[5] Ganesan, G.Sajiv ,Megalan Leo, CIELuv Color Space for Identification and Segmentation of Disease Affected Plant Leaves Using Fuzzy based Approach, IEEE Third International Conference on Science 
Technology Engineering \& Management (ICONSTEM), Chennai,2017,pp.889-894.

.[6] Piyush Chaudhary, Anand K. Chaudhari, Dr. A. N. Cheeran and Sharda Godara, Color Transform Based Approach for Disease Spot Detection on Plant Leaf International Journal of Computer Science and Telecommunications Volume 3, Issue 6, June 2012,pp.65-70..

[7] S. R. N.Neelaveni, "Paddy leaf disease detection classification and remedy finder using $k$ means clustering and image mining," International Journal of Computer Applications, vol. 4, no. 12, 2016, pp. 20 859-20 866.

[8] Radhika Deshmukh, Manjusha Deshmukh, Detection of Paddy Leaf Diseases, International Journal of Computer Applications through International Conference on Advances in Science and Technology Mumbai, 2015 pp.1-3.

[9] A. A. Joshi and B. Jadhav, Monitoring and controlling rice diseases using image processing techniques, in Computing, Analytics and Security Trends (CAST), IEEE International Conference on. Pune ,2016, pp. 471-476. https://doi.org/10.1109/CAST.2016.7915015

[10] Pranjali B. Padol Prof. Anjali A. Yadav SVM Classifier Based Grape Leaf Disease Detection IEEE Conference on Advances in Signal Processing (CASP) Pune,2016,pp. 175-179.

[12] D. Hema and Dr. S. Kannan, Interactive Color Image Segmentation using HSV Color Space, International Journal of Advanced Science and Technology Journal, Volume 7, Issue 1 ,2020,pp.2401-2407.

[13] P. Ganesan, V. Rajini, B. S. Sathish and K. B. Shaik, HSV color space based segmentation of region of interest in satellite images, International Conference on Control, Instrumentation, Communication and Computational Technologies (ICCICCT), Kanyakumari, 2014, pp. 101-105.

[14] García-Lamont, F, Cuevas, A, \& Niño, Y. Segmentation of color images by chromaticity features using self-organizing maps, Ingeniería e Investigación, Volume 36,Issue 2 ,2016, pp. 78-89

[15] R. Amirulah, M. M. Mokji, Z. Ibrahim and U. U. Sheikh, Starfruit Image Segmentation Based on YCbCr Color Space, IEEE Sixth International Conference on Signal-Image Technology and Internet Based Systems, Kuala Lumpur, 2010, pp. 161-164.

[16] Khenilyn P. Lewis,Mary Ann.F.Quioc,Juancho D.Espineli, Image Processing Techniques and Data Mining Algorithms for Coffee Plant's Leaves Classification, International Journal of Advanced Trends in Computer Science and Engineering, Volume 9,Number 2, 2020,pp.1101-1106. https://doi.org/10.30534/ijatcse/2020/31922020

[17] Vikram R,Kalaivani T, Kanimozhi, A Ranjani V,Reena D, Density based Traffic Management System using Image Processing, International Journal of Advanced
Trends in Computer Science and Engineering, Volume 9,Number 2, 2020,pp.997-1001.

https://doi.org/10.30534/ijatcse/2020/15922020

[18] A.Joel Dickson,Dr.J.Arul Linsely,Dr. A. Justin Diraviam, A Study on Segmentation Algorithms for Liver Disorder Analysis in Medical Images, International Journal of Advanced Trends in Computer Science and Engineering ,Volume 9,Number 2, 2020,pp.1159-1165.

https://doi.org/10.30534/ijatcse/2020/40922020 